\title{
MATHEMATICAL MODEL FOR AN AREA SOURCE AND THE POINT SOURCE IN AN URBAN AREA
}

\author{
Pandurangappa $\mathbf{C}^{\mathbf{1}}$, Lakshminarayanachari $\mathrm{K}^{2}$ \\ 1 Professor, Department of Mathematics, RajaRajeswari College of Engineering, India, pandurangappa_c@yahoo.co.in \\ 2 Associate Professor, Department of Mathematics, Sai Vidya Institute of Technology, India, Incharik@yahoo.com
}

\begin{abstract}
A Mathematical model has been developed to study the dispersion of pollutants emitted from an area source and the point source on the boundary in an urban area. The mathematical model has been solved numerically by using the implicit Crank-Nicolson finite difference method. The results of this model have been analysed for the dispersion of air pollutants in the urban area downwind and vertical direction for stable and neutral conditions of the atmosphere in the presence of mesoscale wind. The concentration of pollutants is less in the upwind side of the centre of heat island and more in the downwind side of the centre of heat island in the case of mesoscale wind when compared to without mesoscale wind. In the case of stable atmospheric condition, the maximum concentration of pollutants is observed at the ground surface and near the point source on the boundary. Same phenomenon is observed in neutral atmospheric condition but the magnitude of concentration of pollutants in the neutral atmospheric condition is comparatively less than that of the stable atmospheric condition.
\end{abstract}

Index Terms: Point Source, Area Source, Mesoscale wind

\section{INTRODUCTION}

Pollutants emitted from different surface sources are mixed with the air above the earth's surface. When the concentration of different species of pollutants rises above the respective threshold value then the living species of the environment get affected in several ways preventing their growth and survival. This process of mixing air with harmful substances beyond the threshold value is called air pollution. For different plant and animal species the threshold values of concentrations of the single pollutant are different.

In this paper a numerical model for the atmospheric dispersion of an air pollutant emitted from an area source and a point source on the boundary in the presence of mesoscale wind is described. An area source is an emission source, which is spread out over finite downwind distance. In the absence of removal mechanisms the Gaussian Plume model is the basic method used to calculate the air pollution concentrate from point source (Turner 1970, Carpenter et al 1971, Morgenstern et al 1975). Use of the Gaussian plume model began to receive popularity when Pasquill (1961) published his dispersion rates for plumes over open level terrain. Subsequently, Hilsmeier and Gifford (1962) expressed these estimates in a slightly more convenient, although exactly equivalent, form and this is so called Pasquill-Gifford system for dispersion estimates has been widely used ever since. Runca et al (1975) have presented time dependent numerical model for air pollution due to point source. In this model the boundary conditions at the point source are expressed using a delta function and is approximated numerically by a one-step function having the width $\Delta z_{k}$ (source width) i.e., the source is uniformly distributed on the vertical grid spacing centered at the point source. Arora (1991) used Gaussian distribution for point source on the vertical grid spacing centered at the point source. In this paper the point source is considered arbitrarily on the left boundary of city. The grid points may miss the source because the source is at an arbitrary point. In this case the grid points have to be taken on the source point. To overcome this one can think of the following two methods. One is to use Gaussian distribution for pollutants source at the initial line which is equivalent to the above point source and the other is distributing the point source to its neighbouring two grid points. We have used the second procedure in this numerical model for air pollutants to take into account of a source at an arbitrary point on the left boundary of the city. We have equally distributed the point source to its neighbouring two grid points on boundary of the city. The model has been solved using Crank-Nicolson implicit finite difference technique. Concentration contours are plotted and results are analyzed for various meteorological parameters, and removal mechanisms, with and without mesoscale winds.

\section{MODEL DEVELOPMENT}

The mathematical formulation of the area source air pollution model is based on the conservation of mass equation, which describes advection, turbulent diffusion, chemical reaction, removal mechanisms and emission of pollutants. It is assumed that the terrain is flat, large scale wind velocity is a function of height i.e., $u=u(z)$ and mesoscale wind 
velocities are functions of both distance and height i.e., $W_{e}=W_{e}(z) \quad$ and $u_{e}=u_{e}(x, z)$. The equation for concentration of pollutants can be expressed as follows

$\frac{\partial C}{\partial t}+U \frac{\partial C}{\partial x}+V \frac{\partial C}{\partial y}+W \frac{\partial C}{\partial z}=\frac{\partial}{\partial x}\left(K_{x} \frac{\partial C}{\partial x}\right)+\frac{\partial}{\partial y}\left(K_{y} \frac{\partial C}{\partial y}\right)+\frac{\partial}{\partial z}\left(K_{z} \frac{\partial C}{\partial z}\right)-R C$

where, $\mathrm{C}$ is the pollutant concentration in air at any location $(x, y, z)$ and time $t ; K_{x}, K_{y}$ and $K_{z}$ are the coefficients of eddy diffusivity in the $x, y$ and $z$ directions respectively; $U, V$ and $W$ are the wind velocity components in $x, y$ and $z$ directions respectively and $R$ is the chemical reaction rate coefficient for chemical transformation.

The physical problem consists of an area source which is spread over the surface of the city with finite down wind and infinite cross wind dimensions. We assume that the pollutants are emitted at a constant rate from uniformly distributed area source. The pollutants are transported horizontally by large scale wind which is a function of vertical height $(z)$ and horizontally as well as vertically by local wind caused by urban heat source, called mesoscale wind. We have considered the centre of heat island at a distance $x=l / 2$ i.e., at the centre of the city, also the source region within the urban area which extends to a distance $l$ in the downwind $x$ direction $(0 \leq x \leq$ $l$ ). In this problem we have taken $l=6 \mathrm{~km}$. We compute the concentration distribution till the desired downwind distance $l$ $=6 \mathrm{~km}$ i.e., $0 \leq x \leq l$. The pollutants are considered to be chemically reactive. We assume that the pollutants undergo the removal mechanisms, such as dry deposition, wet deposition, gravitational settling velocity and leakage through the upper boundary. The physical description of the model is shown schematically in figure 1 .

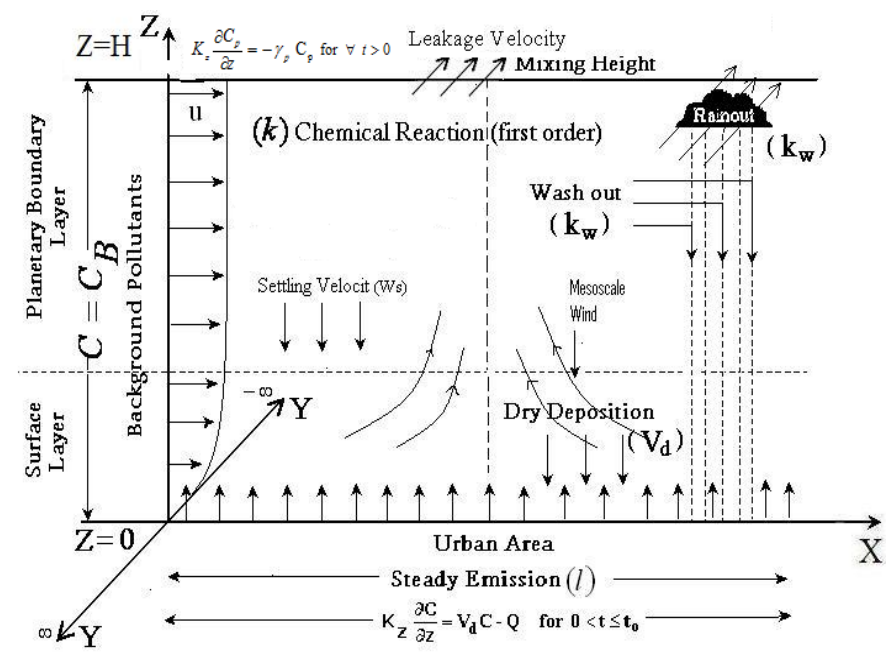

Fig -1: Physical layout of the model.
Further we assume that the

1. Pollutants leak through the top of the boundary i.e. we consider leakage velocity at the top boundary, and

2. Pollutants are chemically reactive, transformation process with first order chemical reaction rate.

3. The lateral flux of pollutants along crosswind direction is assumed to be small i.e., $V \frac{\partial C_{p}}{\partial y}$ and $\frac{\partial}{\partial y}\left(K_{y} \frac{\partial C_{p}}{\partial y}\right) \rightarrow 0$, where $V$ is the velocity in the y direction and $K_{y}$ is the eddydiffusivity coefficient in the y direction.

4. Horizontal advection is greater than horizontal diffusion for not too small values of wind velocity, i.e., meteorological conditions are far from stagnation. The horizontal advection by the wind dominates over horizontal diffusion, i.e., $U \frac{\partial C_{p}}{\partial x}>>\frac{\partial}{\partial x}\left(K_{x} \frac{\partial C_{p}}{\partial x}\right)$, where $U$ and $K_{x}$ are the horizontal wind velocity and horizontal eddy diffusivity along $x$ direction respectively.

Under the above assumptions the governing partial differential equation for the concentration of pollutants is discussed below.

\section{MATHEMATICAL FORMULATION}

The basic governing equation of concentration of pollutants can be written as

$\frac{\partial C_{p}}{\partial t}+U(x, z) \frac{\partial C_{p}}{\partial x}+W(z) \frac{\partial C_{p}}{\partial z}=\frac{\partial}{\partial z}\left(K_{z}(z) \frac{\partial C_{p}}{\partial z}\right)-\left(k+k_{w p}\right) C_{p}$.

where $C_{p}=C_{p}(x, z, t)$ is the ambient mean concentration of pollutant species, $U$ is the mean wind speed in $x$-direction, $W$ is mean wind speed in z-direction, $K_{z}$ is the turbulent eddy diffusivity in $z$-direction, $k_{w p}$ is the first order rainout/washout coefficient of concentration of pollutants $C_{p}$ and $k$ is the first order chemical reaction rate coefficient.

We assume that the region of interest is free from pollution at the beginning of the emission. Thus, the initial condition is

$C_{p}=0$ at $t=0,0 \leq x \leq l$ and $0 \leq z \leq H$,

where $l$ is the length of desired domain of interest in the wind direction and $\mathrm{H}$ is the mixing height. We assume that there is no background pollution of concentration entering at $x=0$ into the domain of interest. Thus

$C_{p}=0$ at $x=0,0 \leq z \leq \mathrm{H}$ and $\forall t>0$.

We assume that the chemically reactive air pollutants are being emitted at a steady rate from the ground level. They are 
removed from the atmosphere by ground absorption and settling velocity. Hence, the corresponding boundary condition takes the form

$$
K_{z} \frac{\partial C_{p}}{\partial z}+W_{s} C_{p}=V_{d p} C_{p}-Q \text { at } z=0,0<x \leq l \quad \forall t>0,
$$

where $\mathrm{Q}$ is the emission rate of pollutant species, $l$ is the source length in the downwind direction, $V_{d p}$ is the dry deposition velocity and $W_{s}$ is the gravitational settling velocity of pollutants. We have considered the source region within the urban area which extends to a distance $l$ in the downwind $x$ direction i.e., the pollutants are assume to be emitted within the city. The pollutants are confined within the mixing height with some amount of leakage across the top boundary of the mixing layer. Thus

$$
K_{z} \frac{\partial C_{p}}{\partial z}=-\gamma_{p} \mathrm{C}_{\mathrm{p}} \text { at } z=H, \quad x>0 \quad \forall t .
$$

\section{METEOROLOGICAL PARAMETERS}

To solve equation (1) we must know realistic form of the variable wind velocity and eddy diffusivity which are functions of vertical distance. The treatment of equation (1) mainly depends on the proper estimation of diffusivity coefficient and velocity profile of the wind near the ground/or lowest layers of the atmosphere. The meteorological parameters influencing eddy diffusivity and velocity profile are dependent on the intensity of turbulence, which is influenced by atmospheric stability. Stability near the ground is dependent primarily upon the net heat flux. In terms of boundary layer notation, the atmospheric stability is characterized by the parameter L (Monin and Obukhov 1954), which is also a function of net heat flux among several other meteorological parameters. It is defined by

$L=-\frac{u_{*}^{3} \rho c_{p} T}{\kappa g H_{f}}$

where $u_{*}$ is the friction velocity, $H_{f}$ the net heat flux, $\rho$ the ambient air density, $\mathrm{c}_{p}$ the specific heat at constant pressure, $T$ the ambient temperature near the surface, $g$ the gravitational acceleration and $\kappa$ the Karman's constant $\approx 0.4 . H_{f}<0$ and consequently $L>0$ represents stable atmosphere, $H_{f}>0$ and $L<0$ represent unstable atmosphere and $H_{f}=0$ and $L \rightarrow \infty$ represent neutral condition of the atmosphere. The friction velocity $u_{*}$ is defined in terms of geostrophic drag coefficient $c_{g}$ and geostrophic wind $u_{g}$ such that

$u_{*}=c_{g} u_{g}$,

where $c_{g}$ is a function of the surface Rossby number $R_{0}=u_{*} / f z_{0}$, where $f$ is the Coriolis parameter due to earth's rotation and $z_{0}$ is the surface roughness length. Lettau
(1959) gave the value of $c_{g n}$, the drag coefficient for a neutral atmosphere in the form

$c_{g n}=\frac{0.16}{\left[\log _{10}\left(R_{0}\right)-1.8\right]}$.

The effect of thermal stratification on the drag coefficient can be accounted through the relations:

$c_{\text {gus }}=1.2 c_{g n}$ for unstable flow,

$c_{g s}=0.8 c_{g n}$ for slightly stable flow and

$c_{g s}=0.6 c_{g n}$ for stable flow.

In order to evaluate the drag coefficient, the surface roughness length $\mathrm{z}_{0}$ may be computed according to the relationship developed by Lettau (1970) i.e., $z_{0}=\bar{H} a / 2 \bar{A}$, where $\bar{H}$ is the effective height of roughness elements, $a$ is the frontal area seen by the wind and $\bar{A}$ is the lot area (i.e., the total area of the region divided by the number of elements).

Finally, in order to connect the stability length $L$ to the Pasquill stability categories, it is necessary to quantify the net radiation index. Ragland (1973) used the following values of $H_{f}$ (Table 2.) for urban area.

Table -1: Net heat flux $H_{f}\left(\right.$ langley $\left.\min ^{-1}\right)$

Net radiating index:4.0 $3.0 \quad 2.0 \quad 1.0 \quad 0.0 \quad-1.0 \quad-2.0$

Net heat flux $H_{f}: 0.24 \quad 0.18 \quad 0.12 \quad 0.06 \quad 0.0 \quad-0.03 \quad 0.06$

\section{EDDY DIFFUSIVITY PROFILES}

Following gradient transfer hypothesis and dimensional analysis, the eddy viscosity, $\mathrm{K}_{\mathrm{M}}$, is defined as

$K_{M}=\frac{u_{*}^{2}}{\partial U / \partial z}$.

Using similarity theory of Monin and Obukhov (1954) the velocity gradient may be written as

$\frac{\partial U}{\partial z}=\frac{u_{*} \phi_{M}}{\kappa z}$.

Substituting this in the equation (9), we have

$K_{M}=\frac{\kappa u_{*} z}{\phi_{M}}$

The function $\phi_{\mathrm{M}}$ depends on $z / L$, where $L$ is MoninObukhov stability length parameter. It is assumed that the surface layer terminates at $\quad z=0.1 \kappa u_{*} / f \quad$ for neutral stability. For stable conditions, surface layer extends to $\mathrm{z}=$ $6 L$.

For the neutral stability condition with $z<0.1 \kappa \boldsymbol{u}_{*} / f$ (within surface layer) we have 
$\phi_{M}=1$ and $K_{M}=\kappa u_{*} z$

For the stable atmospheric flow with $0<z / L<1$ we get

$\phi_{M}=1+\frac{\alpha}{L} z$

and

$$
K_{M}=\frac{\kappa u_{*} z}{1+\frac{\alpha}{\mathrm{L}} z} .
$$

For the stable atmospheric flow with $1<z / L<6$ we have

$\phi_{M}=1+\alpha$ and $K_{M}=\frac{\kappa u_{*} z}{1+\alpha}$.

Webb (1970) has shown that $\alpha=5.2$. In the PBL (planetary boundary layer), where $z / L$ is greater than the limits considered above and $z>0.1 \kappa u_{*} / f$, we have the following expressions for $K_{M}$.

For the neutral atmospheric stability condition with $z>0.1 \kappa u_{*} / f$ we have

$K_{M}=0.1 \kappa^{2} \frac{u_{*}^{2}}{f}$.

For the stable atmospheric flow with $z>6 L$, upto $H$, the mixing height, we have

$$
K_{M}=\frac{6 \kappa u_{*} L}{1+\alpha} \text {. }
$$

Equations (11) to (17) give the eddy viscosity for the conditions needed for the model.

The common characteristics of $\mathrm{K}_{\mathrm{z}}$ is that it has linear variation near the ground, a constant value at mid mixing depth and a decreasing trend as the top of the mixing layer is approached. Shir (1973) gave an expression based on theoretical analysis of neutral boundary layer in the form

$K_{z}=0.4 u_{*} z e^{-4 z / H}$

where $H$ is the mixing height

For stable condition, Ku etal., (1987) used the following form of eddy-diffusivity,

$$
\begin{aligned}
& K_{z}=\frac{\kappa u_{*} z}{0.74+4.7 z / L} \exp (-b \eta), \\
& b=0.91, \quad \eta=z /(L \sqrt{\mu}), \quad \mu=u_{*} /|f L| .
\end{aligned}
$$

The above form of $K_{z}$ was derived from a higher order turbulence closure model which was tested with stable boundary layer data of Kansas and Minnesota experiments. Eddy-diffusivity profiles given by equation (18) and (19) have been used in this model developed for neutral and stable atmospheric conditions.

\section{MESOSCALE WIND}

It is known that in a large city the heat generation causes the rising of air at the centre of the city. Hence the city can be called as heat island. This rising air forms an air circulation and this circulation is completed at larger heights. But we are interested only what happens near the ground level.

In order to incorporate somewhat realistic form of velocity profile in our model which depends on Coriolis force, surface friction, geosrtophic wind, stability characterizing parameter $L$ and vertical height $\mathrm{z}$, we integrate equation (10) from $z_{0}$ to $z+z_{0}$ for stable and neutral conditions. So we obtain the following expressions for wind velocity.

In case of neutral atmospheric stability condition with $z<0.1 \kappa u_{*} / f \quad$ we get

$u=\frac{u_{*}}{\kappa} \ln \left(\frac{z+z_{0}}{z_{0}}\right)$.

It is assumed that the horizontal mesoscale wind varies in the same vertical manner as $u$. The vertical mesoscale wind $W_{e}$ can then be found by integrating the continuity equation and obtain.

$u_{e}=-a\left(x-x_{0}\right) \ln \left(\frac{z+z_{0}}{z_{0}}\right)$,

where $a$ is proportionality constant.

Thus we have

$U(x, z)=u+u_{e}=\left(\frac{u_{*}}{\kappa}-a\left(x-x_{0}\right)\right) \ln \left(\frac{z+z_{0}}{z_{0}}\right)$
$W(z)=w_{e}=a\left[z \ln \left(\frac{z+z_{0}}{z_{0}}\right)-z+z_{0} \ln \left(z+z_{0}\right)\right]$

In case of stable atmospheric condition

For $0<\frac{z}{L}<1$ we get

$u=\frac{u_{*}}{\kappa}\left[\ln \left(\frac{z+z_{0}}{z_{0}}\right)+\frac{\alpha}{L} z\right]$.

$u_{e}=-a\left(x-x_{0}\right)\left[\ln \left(\frac{z+z_{0}}{z_{0}}\right)+\frac{\alpha}{L} z\right]$

$U(x, z)=u+u_{e}=\left(\frac{u_{*}}{\kappa}-a\left(x-x_{0}\right)\right)\left[\ln \left(\frac{z+z_{0}}{z_{0}}\right)+\frac{\alpha}{L} z\right]$

$W(z)=w_{e}=a\left[z \ln \left(\frac{z+z_{0}}{z_{0}}\right)-z+z_{0} \ln \left(z+z_{0}\right)+\frac{\alpha}{2 L} z^{2}\right]$

For $1<\frac{z}{L}<6$ we get 
$u=\frac{u_{*}}{\kappa}\left[\ln \left(\frac{z+z_{0}}{z_{0}}\right)+5.2\right]$.

$u_{e}=-a\left(x-x_{0}\right)\left[\ln \left(\frac{z+z_{0}}{z_{0}}\right)+5.2\right]$

$U(x, z)=u+u_{e}=\left(\frac{u_{*}}{\kappa}-a\left(x-x_{0}\right)\right)\left[\ln \left(\frac{z+z_{0}}{z_{0}}\right)+5.2\right]$

$W(z)=w_{e}=a\left[z \ln \left(\frac{z+z_{0}}{z_{0}}\right)+z_{0} \ln \left(z+z_{0}\right)+4.2 z\right]$

In the planetary boundary layer $((Z / L) \geq 6)$, above the surface layer, power law scheme has been employed.

$$
\begin{aligned}
& u=\left(u_{g}-u_{s l}\right)\left(\frac{z-z_{s l}}{H-z_{s l}}\right)^{p}+u_{s l}, \\
& u_{e}=-a\left(x-x_{0}\right)\left[\left(\frac{z-z_{s l}}{H-z_{s l}}\right)^{p}+u_{s l}\right] \\
& U(x, z)=u+u_{e} \\
& =\left[\left(u_{g}-u_{s l}\right)-a\left(x-x_{0}\right)\right]\left(\frac{z-z_{s l}}{H-z_{s l}}\right)^{p}+\left(1-a\left(x-x_{0}\right)\right) u_{s l} \\
& W(z)=w_{e}=a\left[\left(u_{g}-u_{s l}\right) \frac{\left(z-z_{s l}\right)}{p+1}\left(\frac{z-z_{s l}}{H-z_{s l}}\right)^{p}+z u_{s l}\right]
\end{aligned}
$$

where, $u_{g}$ is the geostrophic wind, $z_{s l}$ the top of the surface layer, $x_{0}$ is the $x$ co-ordinate of centre of heat island, $\mathrm{H}$ is the mixing height and $p$ is an exponent which depends upon the atmospheric stability. Jones et al., (1971) suggested the values for the exponent $p$, obtained from the measurements made from urban wind profiles, as follows:

$$
p= \begin{cases}0.2 & \text { for neutral conditions } \\ 0.35 & \text { for slightly stable flow } \\ 0.5 & \text { for stable flow }\end{cases}
$$

Wind velocity profiles given by equations (20), (24), (28) and (32) are due to Ragland (1973) and (22), (23), (26), (27), (30), (31), (34) and (35) are modified as per Dilley -Yen (1971) are used in this model.

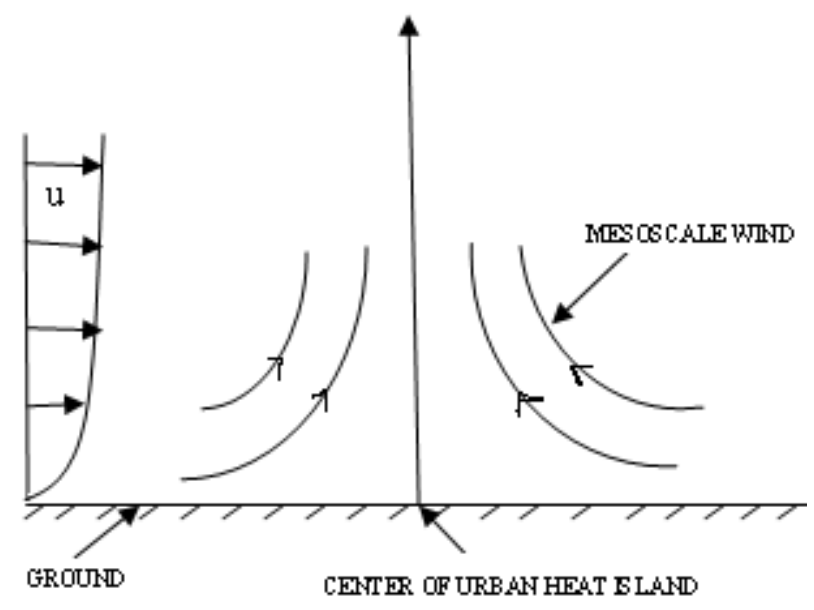

Fig -2: A simulated urban heat island with the large scale and mesoscale winds.

The above wind velocity profiles are valid only for $(u * / \kappa)-a\left(x-x_{0}\right)>0$. This relation puts a limit on $x$. When $\mathrm{x}$ approaches $(u * / a \kappa)+x_{0}$ the Stream lines are asymptotically vertical and there will be no transfer of material across the vertical plane $x=(u * / a \kappa)+x_{0}$. Therefore the wind velocity profiles are valid only for $x \leq\left(u_{*} / a \kappa\right)+x_{0}$. Thus the range of validity increases indefinitely as mesoscale wind decreases. In this model we have taken the mesoscale wind parameter $a=0.00004$.

\section{NUMERICAL METHOD}

Equation (1) are solved numerically using the implicit CrankNicolson finite difference method in this paper. We note that it is difficult to obtain the analytical solution for equation (1) because of the complicated form of wind speed $\mathrm{U}(\mathrm{x}, \mathrm{z})$ and eddy diffusivity $\mathrm{K}_{\mathrm{z}}(\mathrm{z})$ considered in this paper (see sections 56). Hence, we have used numerical method based on CrankNicolson finite difference scheme to obtain the solution. The detailed numerical method and procedure to solve the partial differential equation (1) is described below (Roache 1976, John, F. Wendt 1992).

The governing partial differential equation (1) is

$$
\begin{aligned}
\frac{\partial C_{p}}{\partial t}+U(x, z) \frac{\partial C_{p}}{\partial x}+W(z) \frac{\partial C_{p}}{\partial z} \\
=\frac{\partial}{\partial z}\left(K_{z}(z) \frac{\partial C_{p}}{\partial z}\right)-\left(k+k_{w p}\right) C_{p} .
\end{aligned}
$$

Now this equation is replaced by the equation valid at time step $n+1 / 2$ and at the interior grid points $(i, j)$. The spatial derivatives are replaced by the arithmetic average of its finite difference approximations at the $n^{t h}$ and $(n+1)^{t h}$ time steps and we replace the time derivative with a central difference 
with time step $n+1 / 2$. Then equation (1) at the grid points $(i, j)$ and time step $n+1 / 2$ can be written as

$$
\begin{array}{r}
\left.\frac{\partial C_{p}}{\partial t}\right|_{i j} ^{n+\frac{1}{2}}+\frac{1}{2}\left[\left.U(x, z) \frac{\partial C_{p}}{\partial x}\right|_{i j} ^{n}+\left.U(x, z) \frac{\partial C_{p}}{\partial x}\right|_{i j} ^{n+1}\right] \\
+\frac{1}{2}\left[\left.W(z) \frac{\partial C_{p}}{\partial z}\right|_{i j} ^{n}+\left.W(z) \frac{\partial C_{p}}{\partial z}\right|_{i j} ^{n+1}\right] \\
=\frac{1}{2}\left[\left.\frac{\partial}{\partial z}\left(K_{z}(z) \frac{\partial C_{p}}{\partial z}\right)\right|_{i j} ^{n}+\left.\frac{\partial}{\partial z}\left(K_{z}(z) \frac{\partial C_{p}}{\partial z}\right)\right|_{i j} ^{n+1}\right] \\
-\frac{1}{2}\left(k+k_{w p}\right)\left(C_{p i j}^{n}+C_{p i j}^{n+1}\right),
\end{array}
$$

for each $i=2,3,4, \ldots . i \max , j=2,3,4, \ldots . j \max -1, n=0,1,2 \ldots$.

Using

$$
\begin{aligned}
& \left.\frac{\partial C_{p}}{\partial t}\right|_{i j} ^{n+\frac{1}{2}}=\frac{C_{p i j}^{n+1}-C_{p i j}^{n}}{\Delta t}, \\
& \left.U(x, z) \frac{\partial C_{p}}{\partial x}\right|_{i j} ^{n}=U_{i j}\left[\frac{C_{p i j}^{n}-C_{p i-1 j}^{n}}{\Delta x}\right] \\
& \left.U(x, z) \frac{\partial C_{p}}{\partial x}\right|_{i j} ^{n+1}=U_{i j}\left[\frac{C_{p i j}^{n+1}-C_{p i-1 j}^{n+1}}{\Delta x}\right] \\
& \left.W(z) \frac{\partial C_{p}}{\partial z}\right|_{i j} ^{n}=W_{j}\left[\frac{C_{p i j}^{n}-C_{p i-1 j}^{n}}{\Delta z}\right] \\
& \left.W(z) \frac{\partial C_{p}}{\partial z}\right|_{i j} ^{n+1}=W_{j}\left[\frac{C_{p i j}^{n+1}-C_{p i-1 j}^{n+1}}{\Delta z}\right] \\
& \left.\frac{\partial}{\partial z}\left(K_{z}(z) \frac{\partial C_{p}}{\partial z}\right)\right|_{i j} ^{n} \\
& =\frac{1}{2(\Delta z)^{2}}\left[\left(K_{j+1}+K_{j}\right)\left(C_{p i j+1}^{n}-C_{p i j}^{n}\right)-\left(K_{j}+K_{j-1}\right)\left(C_{p i j}^{n}-C_{p i j-1}^{n}\right)\right] \\
& \left.\frac{\partial}{\partial z}\left(K_{z}(z) \frac{\partial C_{p}}{\partial z}\right)\right|_{i j} ^{n+1} \\
& =\frac{1}{2(\Delta z)^{2}}\left[\left(K_{j+1}+K_{j}\right)\left(C_{p i j+1}^{n+1}-C_{p i j}^{n+1}\right)-\left(K_{j}+K_{j-1}\right)\left(C_{p i j}^{n+1}-C_{p i j-1}^{n+1}\right)\right]
\end{aligned}
$$

Equation (36) can be written as

$$
B_{j} C_{p i j-1}^{n+1}+D_{i j} C_{p i j}^{n+1}+E_{j} C_{p i j+1}^{n+1}
$$

$$
=F_{i j} C_{p i-i j}^{n}+G_{j} C_{p i j-1}^{n}+M_{i j} C_{p i j}^{n}+N_{j} C_{p i j+1}^{n}-A_{i j} C_{p i-i j}^{n+1},
$$

for each $i=2,3,4, \ldots . . i \max$, for each $j=2,3,4, \ldots \ldots . j m a x-1$ and $n=0,1,2,3, \ldots \ldots$

Here

$$
\begin{aligned}
& A_{i j}=-U_{i j} \frac{\Delta t}{2 \Delta x}, \quad F_{i j}=U_{i j} \frac{\Delta t}{2 \Delta x}, \\
& B_{j}=-\left[\frac{\Delta t}{4 \Delta z^{2}}\left(K_{j}+K_{j-1}\right)+\frac{\Delta t}{2 \Delta z} W_{j}\right], \\
& G_{j}=\left[\frac{\Delta t}{4 \Delta z^{2}}\left(K_{j}+K_{j-1}\right)+\frac{\Delta t}{2 \Delta z} W_{j}\right], \\
& E_{j}=-\frac{\Delta t}{4 \Delta z^{2}}\left(K_{j}+K_{j+1}\right), \quad N_{j}=\frac{\Delta t}{4 \Delta z^{2}}\left(K_{j+1}+K_{j}\right), \\
& D_{i j}=1+\frac{\Delta t}{2 \Delta x} U_{i j}+\frac{\Delta t}{2 \Delta z} W_{j} \\
& \quad+\frac{\Delta t}{4 \Delta z^{2}}\left(K_{j+1}+2 K_{j}+K_{j-1}\right)+\frac{1}{2}\left(k+k_{w p}\right), \\
& M_{i j}=1-\frac{\Delta t}{2 \Delta x} U_{i j}-\frac{\Delta t}{2 \Delta z} W_{j} \\
& \quad-\frac{\Delta t}{4 \Delta z^{2}}\left(K_{j+1}+2 K_{j}+K_{j-1}\right)-\frac{1}{2}\left(k+k_{w p}\right) .
\end{aligned}
$$

and imax is the $i$ value at $x=l$ and jmax is the value of $\mathrm{j}$ at $z=H$.

The initial condition (2) can be written as

$$
C_{p i j}^{0}=0 \quad \text { for } \quad j=1,2, \ldots \ldots \ldots . . j \max , i=1,2, \ldots \ldots \ldots . . i \max
$$

The point source is considered arbitrarily on the left boundary of the city. The grid points may miss the source because the source is at an arbitrary point. To overcome this one can think of the following two methods. One is to use Gaussian distribution for pollutants source at the initial line which is equivalent to the above point source and the other is distributing the point source to its neighbouring two grid points. We have used the second procedure in this numerical model for air pollutants to take into account of a source at an arbitrary point on the $z$-axis. We have equally distributed the point source to its neighbouring two grid points on $z$-axis i.e., at the beginning of the urban city. The condition (3) becomes

$$
\begin{gathered}
C_{p i j}^{n+1}=\frac{Q_{1}}{2 U(i, j)} \text { for } i=1, j=j s, j s+1 n=0,1,2, \ldots \\
C_{p i j}^{n+1}=0 \text { for } i=1, j=1,2,3, \ldots j s-1, j s+2, j s+3, \ldots j \max \\
n=0,1,2, \ldots \ldots . .
\end{gathered}
$$

The boundary condition (4) can be written as

$$
\left(1+\left(V_{d}+W_{s}\right) \frac{\Delta z}{K_{j}}\right) C_{p i j}^{n+1}-C_{p i j+1}^{n+1}=Q \frac{\Delta z}{K_{j}},
$$

for $j=1, i=2,3,4, \ldots \ldots$. imax and $n=0,1,2,3 \ldots$. 
The boundary condition (5) can be written as

$$
\left(1+\left(\gamma_{p}\right) \frac{\Delta z}{K_{j}}\right) C_{p i j}^{n+1}-C_{p i j-1}^{n+1}=0,
$$

for $j=$ jmax, $i=2,3,4 \ldots .$, imax

In the equation (44) a term with unknown $C_{p i-1 j}^{n+1}$ is taken to right hand side. Normally all unknown terms should be on the left hand side and the system of equations should be solved together with $C_{p i-1 j}^{n+1}$. In that case the system of equations will not be tridiagonal system. Therefore Thomas algorithm cannot be applied.

To overcome this we transfer the term with unknown $C_{p i-1 j}^{n+1}$ to right hand side as seen in equation (44) and solve the system first for $i=2$ and $j=2,3,4, \ldots \ldots . j \max -1$. Now $C_{p i-1 j}^{n+1}$ values are known from the boundary condition (44a) and hence it is known. Hence, all the terms on the right hand side are known. Now the system of equations (44) for $i=2$ and $j=2,3,4, \ldots \ldots . . j \max -1$ along with the relevant boundary conditions (45) for $i=2$ and $j=1$ and (46) for $i=2$ and $j=j \max$ become tridiagonal structure which is solved using Thomas algorithm. Now the system (44) for $i=3$ and $j=2,3,4, \ldots \ldots . j \max -1$ along with the relevant boundary conditions is solved. Now the values of $C_{p i-1 j}^{n+1}$ i.e., $C_{p 2 j}^{n+1}$ on the right hand side is known from the previous step. Hence the system still remains tridiagonal and solved using Thomas algorithm. This argument can also be extended for each of the remaining values of $i=4,5,6, \ldots \ldots . . i \max$. Thus although the system equations (44) with boundary conditions appears that it is not tridiagonal, because of boundary condition it becomes tridiagonal and the solution of the system is obtained using Thomas algorithm.

\section{RESULTS AND DISCUSSIONS}

A numerical model for computation of the ambient air concentration of pollutants along down-wind and vertical directions emitted from an area source along with point source on the boundary $\left(x=0\right.$ and $\left.z=z_{s}\right)$ with removal mechanisms, mesoscale wind and transformation process has been presented. The numerical model permits the estimation of concentration distribution for more realistic meteorological conditions. An area source is an emission source which is spread out over the surface of the city with finite down wind and infinite cross wind dimensions where major source being vehicular emissions due to traffic flow. In addition to area source being mainly vehicular exhausts due to traffic flow, we have considered a point source at $x=0$ and height $z=z_{s}$ i.e., at the left boundary of the city. Since the point source is assumed to be at an arbitrary point on the boundary and the grid lengths are arbitrarily chosen in conformity with the numerical scheme there may not be any grid point coinciding with the position of point source. In that case the numerical scheme will fail to sense the existence of a point source. To overcome this difficulty one can use an equivalent Gaussian distribution of pollutants along the $z$-axis in place of a point source. One can also use the other method where the source strength is distributed equally on the neighboring points of the point source. In this paper we have adopted the latter procedure. In the present problem the pollutant strength is equally distributed on the two neighboring points on the $z$ axis. If $Q_{1}$ is the strength of the point source then we have taken $Q_{1} / 2$ on each of the neighboring points on the $z$-axis. We have considered the desired domain of length $l=6 \mathrm{~km}$ and mixing height $624 \mathrm{~m}$. We have considered grid size $75 \mathrm{~m}$ along $x$-direction and $1 \mathrm{~m}$ along $z$ direction. The pollutants are chemically reactive and we have considered source region extending up to $l=6 \mathrm{~km}$.

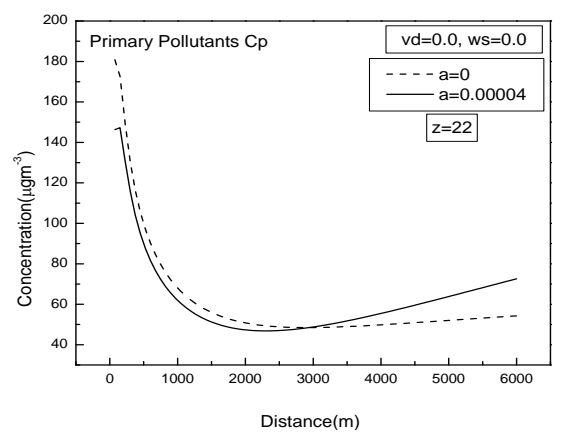

Fig -3: Concentration versus Distance of pollutants with and without mesoscale wind for stable case.

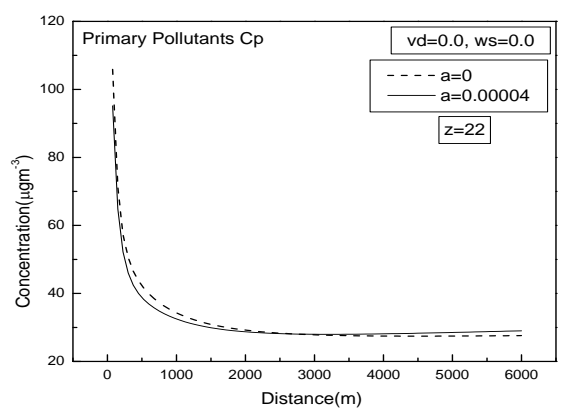

Fig -4: Concentration versus distance of pollutants with and without mesoscale wind for neutral case.

In figures 3 and 4 the effect of mesoscale wind on pollutants for stable and neutral cases without removal mechanisms is studied. The concentration of pollutants is less on the upwind side of centre of heat island $(x=3 \mathrm{~km})$ and more on the downwind side of centre of heat island in the presence of mesoscale wind $(a=0.00004)$ compared to that in the 
absence of mesoscale wind $(a=0.0)$. This is due to the horizontal component of mesoscale wind which is along the large scale wind on the left and against on the right.

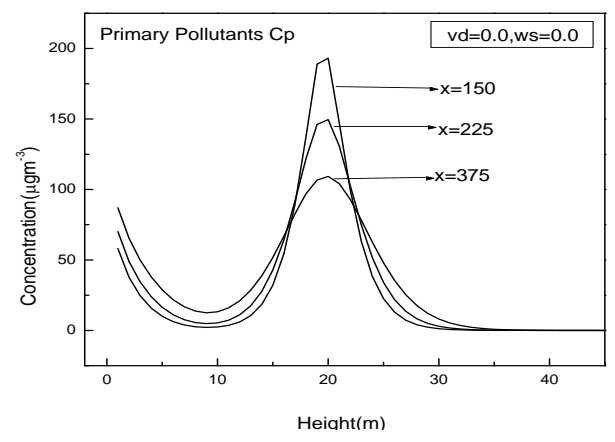

Fig -5: Concentration versus height pollutants without removal mechanisms for stable case

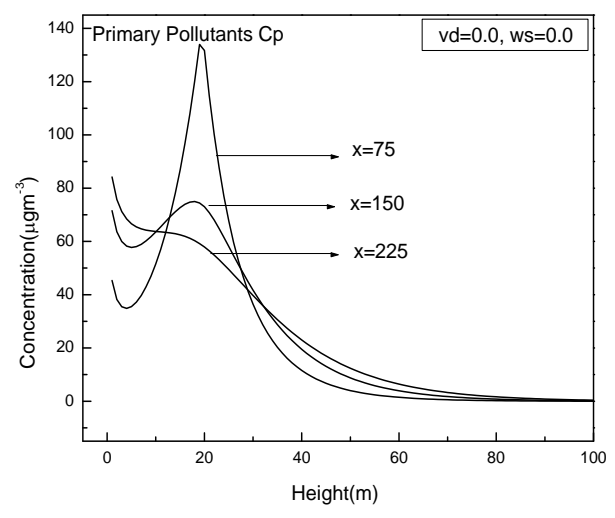

Fig -6: Concentration versus height of pollutants without removal mechanisms for neutral case

Figures 5 and 6 demonstrate that the concentration versus height at different distances of pollutants for stable and neutral cases without removal mechanisms. We have considered a point source at $x=0$ and height $z=20.5 \mathrm{~m}$ along with area source up to the urban city length $l=6 \mathrm{~km}$. From the figure we find that the concentration of the pollutant is high near the ground level due to the area source. As height increases the pollutant concentration decreases up to the height $10 \mathrm{~m}$ and then increases up to around $20.5 \mathrm{~m}$ reaching maximum there, since we have considered the point source being at the height $20.5 \mathrm{~m}$. As we move towards the downwind direction the peak value of the concentration at $20.5 \mathrm{~m}$ height decreases. Similar effect is observed in neutral atmospheric condition since the neutral case enhances the vertical mixing, the concentration of primary and secondary pollutants reaches increased heights compared to that in the case of stable atmospheric condition.

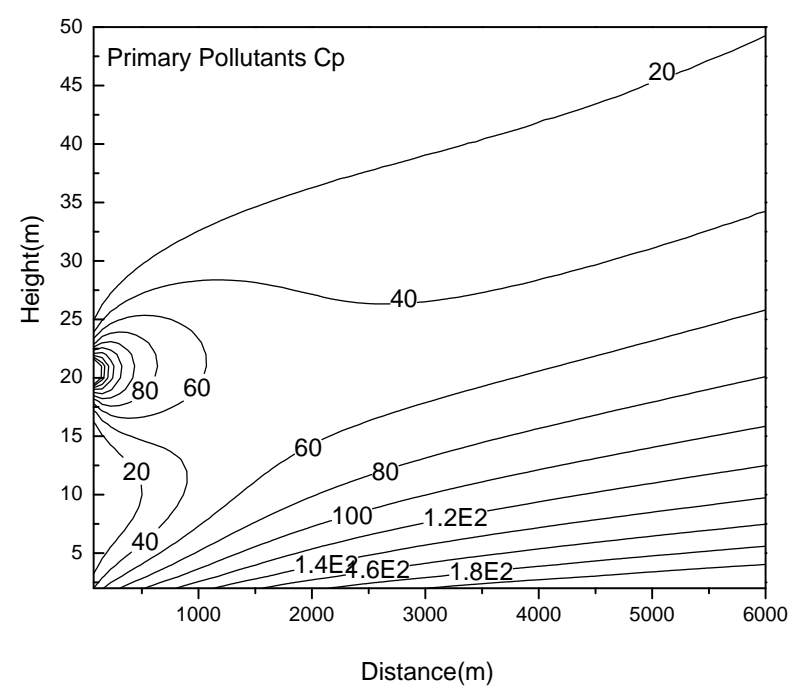

Fig -7: Concentration contours of pollutants for stable case

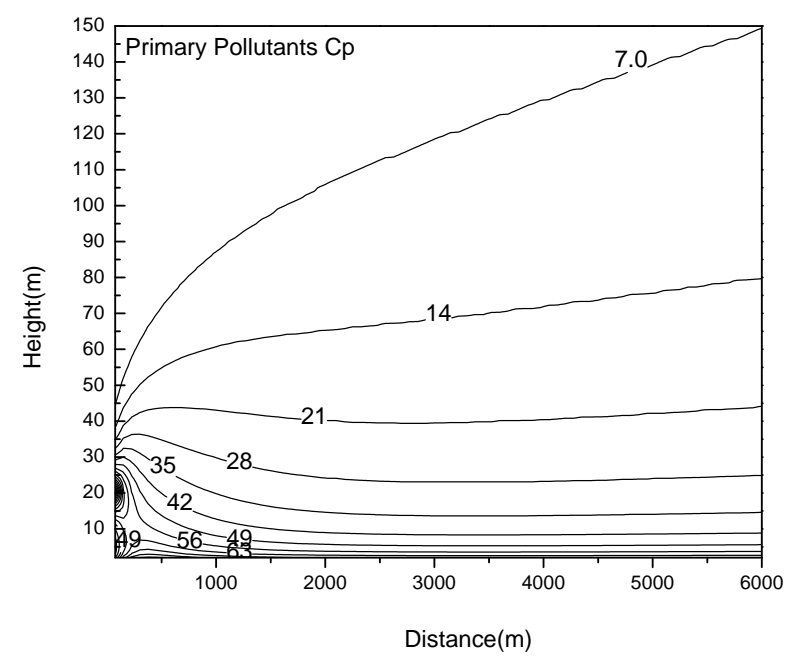

Fig -8: Concentration contours of pollutants for neutral case

The concentration contours of pollutants are drawn in figures 7 and 8 for both stable and neutral cases. We observe that the concentration of pollutants is more at the ground level $(z=2 m)$, at the end of the city region $(x=6000 m)$ and near the point source $\left(x_{s}=0\right.$ and $z_{s}=20.5 \mathrm{~m}$ ). This is because we have considered the area source at the ground level, the advection is in the downwind direction and the point source at $x=0$ and height $\mathrm{z}=20.5 \mathrm{~m}$ i.e., at the beginning of the city. The magnitude of pollutants concentration is higher in stable case and is lower in the neutral case. This is because neutral case enhances vertical diffusion to greater heights and thus the concentration is less. 


\section{CONCLUSIONS}

The effect of mesoscale wind on a two dimensional mathematical model of air pollution due to area source along with a point source on the boundary is presented to simulate the dispersion processes of gaseous air pollutants in an urban area in the presence of mesoscale wind. A numerical model for the computation of the ambient air pollutants concentration emitted from an urban area source along with a point source on the boundary undergoing various removal mechanisms and transformation process is presented. The results of this model have been analysed for the dispersion of air pollutants in the urban area downwind and vertical direction for stable and neutral conditions of the atmosphere in the presence of mesoscale wind. The concentration of pollutants is less in the upwind side of the centre of heat island and more in the downwind side of the centre of heat island in the case of mesoscale wind when compared to without mesoscale wind. This is due to increase of velocity in the upwind direction and decrease in the downwind direction of the centre of heat island by the mesoscale wind. We notice that as removal mechanisms increase the concentration of pollutants decreases. In the case of stable atmospheric condition, the maximum concentration of pollutants is observed at the ground surface and near the point source. Also as the chemical rate reaction coefficient increases the concentration of secondary pollutant increases. Same phenomenon is observed in neutral condition but the magnitude of concentration of pollutants in the neutral condition is comparatively less than that of the stable atmospheric condition.

\section{REFERENCES:}

[1]. Turner, D. B. 1970. Work book of Atmospheric dispersion estimates. USEPA Report AP-26. US. Govt. Printing office, Washington DC.

[2]. Carpenter, S. B., Montogmery, T.L., Deavitt, J.M., Colbough, W.D. and Thomas, F.W. 1971 Principal Plume dispersion models: TVA powerplants. J. Air Pollut. Control Ass. 21(8), 491.

[3]. Morgenstern P., Morgenstern L. N., Chang K. M., Barrett D. H. and Mears C. 1975 Modelling analysis of power plants for compliance extensions in 51 air quality control regions. J. Air Pollut. Control Ass. 25(3). 287-291.

[4]. Pasquill, F. 1961 The estimation of dispersion of windborne material. Meteorol Mag. 90, 33.

[5]. Hilsmeir, W. and Gifford, F. 1962 Graphs for estimating atmospheric dispersion. USAEC, Division of Technical Information, ORD-549.

[6]. Runca, E. and Sardei, F. 1975 Numerical treatment of time dependent advection and diffusion of air pollutants. Atmos. Environ., 9, 69-80

[7]. Arora, U., Gakkar S. and Guptha R.S., 1991. Removal model suitable for air pollutants emitted from an elevated source. Appl. Math. Modelling, 15, 386-389,
[8]. Monin, A. S., Obukhov, A. M. 1954 Basic laws of turbulent mixing in the ground layer of the atmosphere. Dokl. Akad. SSSR, 151, 163.

[9]. Lettau, H. H. 1959 Wind profile, surface stress and geostrophic drag coefficients in the atmospheric surface layer. Advances in Geophysis, Academic Press, New York, 6, 241.

[10]. Lettau, H. H. 1970 Physical and meteorological basis for mathematical models of urban diffusion processes. Proceedings of symposium on Multiple Source Urban Diffusion Models, USEPA Publication AP-86.

[11]. Ragland, K. W. 1973 Multiple box model for dispersion of air pollutants from area sources. Atmospheric Environment 7, 1071.

[12]. Webb, E. K. 1970 Profile relationships: the long-linear range and extension to strong stability. Quart. J. R. Met. Soc. 96, 67.

[13]. Shir, C. C 1973 A preliminary numerical study of a atmospheric turbulent flows in the idealized planetary boundary layer. J. Atmos. Sci. 30, 1327.

[14]. Ku, J. Y., Rao, S. T. and Rao, K. S. 1987 Numerical simulation of air pollution in urban areas: Model development, 21 (1), 201.

[15]. Jones, P. M. Larrinaga M, A. B and Wilson, C. B. 1971. The urban wind velocity profile. Atmospheric Environment 5, 89-102.

[16]. Dilley, J.F, Yen, K.T., 1971. Effect of mesoscale type wind on the pollutant distribution from a line source, Atmospheric Environment Pergamon, 5, pp 843-851.

[17]. Roache, P. J. 1976 Computational fluid dynamics. Hermosa publications.

[18]. John, F. Wendt. 1992 Computational fluid dynamics-An introduction (Editor) A Von Karman Institute Book SpringerVerlag

\section{BIOGRAPHIES}

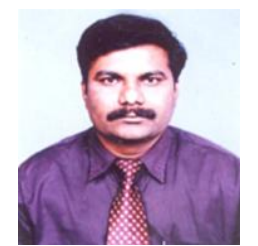

Pandurangappa $\mathbf{C}$ has obtained M.Sc. degree (1998) and Ph.D. degree (2010) from Bangalore University. He is having more than 12 years of teaching experience. Presently he is working as a Professor and Head in the department of Mathematics at Raja Rajeshwari College of Engineering, Bangalore. His area of interest is atmospheric Science and has published several papers in Journals of National/International repute.

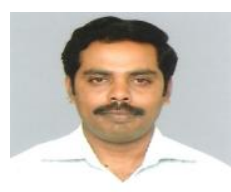

Lakshminarayanachari $\mathbf{K}$ has obtained M.Sc. degree (2000) and Ph.D. degree (2008) from Bangalore University. He is having more than 10 years of teaching experience. Presently he is working as an Associate Professor in the department of Mathematics at, Sai Vidya Institute of Technology, Bangalore. His area of interest is atmospheric science and has published several papers in Journals of National/International repute 